University of Nebraska - Lincoln

DigitalCommons@University of Nebraska - Lincoln

4-1-1998

\title{
Crossover from pure Ising to random-exchange dominated behavior of the two-dimensional antiferromagnet $\mathrm{Rb}_{2} \mathrm{Co}_{1-x} \mathrm{Mg}_{\mathrm{x}} \mathrm{F}_{4}$
}

\author{
Christian Binek \\ University of Nebraska-Lincoln, cbinek@unl.edu \\ Wolfgang Kleemann \\ University Duisburg-Essen, Duisburg, Germany, wolfgang.kleemann@uni-due.de \\ David P. Belanger \\ University of California, Santa Cruz, daveph@ucsc.edu
}

Follow this and additional works at: https://digitalcommons.unl.edu/physicsbinek

Part of the Physics Commons

Binek, Christian; Kleemann, Wolfgang; and Belanger, David P., "Crossover from pure Ising to randomexchange dominated behavior of the two-dimensional antiferromagnet $\mathrm{Rb}_{2} \mathrm{Co}_{1-x} \mathrm{Mg}_{x} \mathrm{~F}_{4}$ " (1998). Christian Binek Publications. 10.

https://digitalcommons.unl.edu/physicsbinek/10

This Article is brought to you for free and open access by the Research Papers in Physics and Astronomy at DigitalCommons@University of Nebraska - Lincoln. It has been accepted for inclusion in Christian Binek Publications by an authorized administrator of DigitalCommons@University of Nebraska - Lincoln. 


\title{
Crossover from pure Ising to random-exchange dominated behavior of the two-dimensional antiferromagnet $\mathrm{Rb}_{2} \mathrm{Co}_{1-x} \mathrm{Mg}_{x} \mathrm{~F}_{4}$
}

\author{
Ch. Binek and W. Kleemann \\ Angewandte Physik, Gerhard-Mercator-Universität Duisburg, D-47048 Duisburg, Germany \\ D. P. Belanger \\ Department of Physics, University of California, Santa Cruz, California 95064
}

(Received 1 October 1997)

\begin{abstract}
The temperature dependence of the uniform susceptibility $\chi$ of diluted two-dimensional Ising antiferromagnets $\mathrm{Rb}_{2} \mathrm{Co}_{1-x} \mathrm{Mg}_{x} \mathrm{~F}_{4}, 0 \leqslant x \leqslant 0.4$, is investigated in the limit of vanishing external field. Data for $x=0.15$ are compared with those obtained for $x=0$ and 0.4 by Breed et al. (1969) and Ikeda (1983), respectively. Whereas in the pure case, $x=0$, Fisher's (1962) “energetic contribution" dominates, Aharony et al.'s $(1979,1986)$ "random contribution"' becomes increasingly important with increasing $x$. Taking into account both terms not only with respect to the global ordering temperature $T_{N}(x)$, but also in relation to the "local" phase transition temperatures $T_{N}^{\prime}$ throughout the Griffiths regime, $T_{N}(x) \leqslant T_{N}^{\prime} \leqslant T_{N}(0)$, a satisfactory quantitative description of $\chi$ vs $T$ is deduced for any $x$ above the percolation threshold. [S0163-1829(98)05914-1]
\end{abstract}

\section{INTRODUCTION}

Although the temperature dependence of the uniform zero-field susceptibility of the pure two-dimensional (2D) Ising antiferromagnet has extensively been studied by Fisher et al., ${ }^{1,2}$ there is, to the best of our knowledge, no convincing attempt to describe its overall temperature dependence in diluted 2D Ising antiferromagnets. It is the aim of this article to give a quantitative description of the overall temperature dependence of the uniform magnetic zero-field susceptibility of $\mathrm{Rb}_{2} \mathrm{Co}_{1-x} \mathrm{Mg}_{x} \mathrm{~F}_{4}$ and its dilution dependent evolution. It turns out, that two aspects are of major importance. On the one hand, it is the dilution induced crossover from pure Ising to random-exchange behavior. Here the relevance of Griffiths-type weak singularities ${ }^{3}$ has to be considered. As was shown qualitatively in the case of the diluted 3D Ising antiferromagnet $\mathrm{Fe}_{1-x} \mathrm{Zn}_{x} \mathrm{~F}_{2}, x=0.53,{ }^{4}$ anomalous behavior of the uniform susceptibility is expected in the temperature range $T_{N}(x) \leqslant T \leqslant T_{N}(0)$.

In the case of the $2 \mathrm{D}$ system $\mathrm{Rb}_{2} \mathrm{Co}_{1-x} \mathrm{Mg}_{x} \mathrm{~F}_{4}$, the anomalies due to singular behavior at "local" phase transitions turn out to be directly measurable throughout the above Griffiths range. On the other hand and in addition, with increasing dilution $x$ disorder-induced contributions to the uniform susceptibility become increasingly important. According to Aharony and co-workers ${ }^{5,6}$ these terms are due to the local imbalance of the sublattice occupations, which arise below the ordering temperature $T_{N}(x)$. In this paper evidence is provided that this conjecture also holds for the entire series of "local" phase transitions at $T_{N}^{\prime}$ within the Griffiths phase, $T_{N}(x) \leqslant T_{N}^{\prime} \leqslant T_{N}(0)$. It turns out that these contributions are at the origin of the surprisingly large extra peak that is observed in the vicinity of $T_{N}(x)$ at sufficiently large dilution, e.g., $x=0.4 .^{7}$

\section{EXPERIMENTAL RESULTS}

Single crystals of pure $\mathrm{Rb}_{2} \mathrm{CoF}_{4}$ are known as prototypical 2D Ising antiferromagnets. ${ }^{8}$ Their $2 \mathrm{D}$ magnetic character originates in the double perovskite structure of the $\mathrm{K}_{2} \mathrm{NiF}_{4}$ type. In contrast with the strong antiferromagnetic (af) inplane coupling $J$ of the $\mathrm{Co}^{2+}$ ions, there is only weak af coupling $J^{\prime}$ between adjacent layers $\left(J^{\prime} / J \approx 10^{-6}\right)$. The corresponding 2D-af long-range order sets in at $T \leqslant T_{N}$ $=102 \mathrm{~K}$. Simultaneously, owing to dimensional crossover, 3D long-range order is established.

Figure 1 shows the temperature dependence of the magnetic uniform susceptibility of pure $\mathrm{Rb}_{2} \mathrm{CoF}_{4}$ (full circles). The data were measured by Breed and co-workers using a pendulum magnetometer. ${ }^{8}$ The static susceptibility $\chi$ $=\partial M / \partial H$ was approximately determined according to $\chi$ $\approx M / H$ where $M$ is the magnetization and $H$ is a small applied magnetic field of the strength $H=0.77 \mathrm{MA} / \mathrm{m}(9640$ $\mathrm{Oe})$. The well-known temperature dependence of the susceptibility of the pure $\mathrm{Rb}_{2} \mathrm{CoF}_{4}$ gives rise to the logarithmic singularity of $d(T \chi) / d T$ vs $T$ at $T_{N} \approx 102 \mathrm{~K}$ (Ref. 2) (Fig. 1,

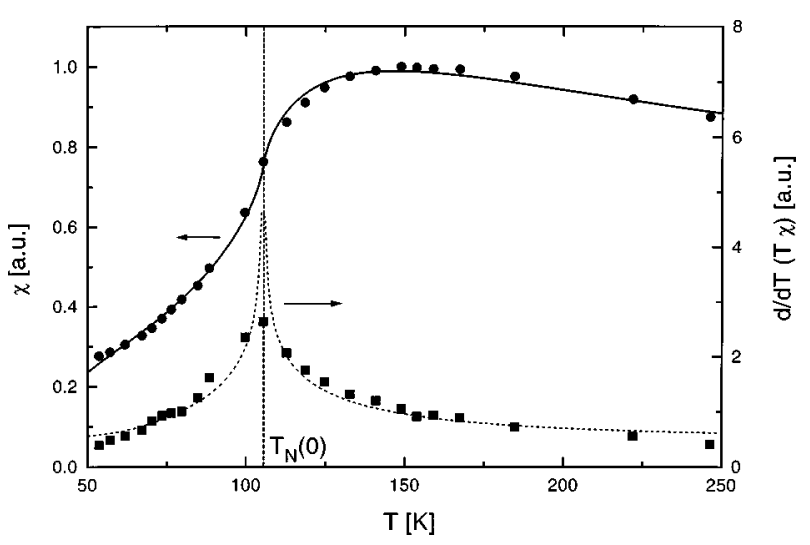

FIG. 1. Temperature dependence of the zero field susceptibility $\chi$ (circles) of $\mathrm{Rb}_{2} \mathrm{CoF}_{4}$ (data from Ref. 8) and the corresponding derivative of $T \chi$ with respect to temperature (squares). The solid and dashed lines represent the best fit of Eq. (11) to the data and its temperature derivative, respectively (see text). $T_{N}(0)$ is indicated by a vertical dashed line. 


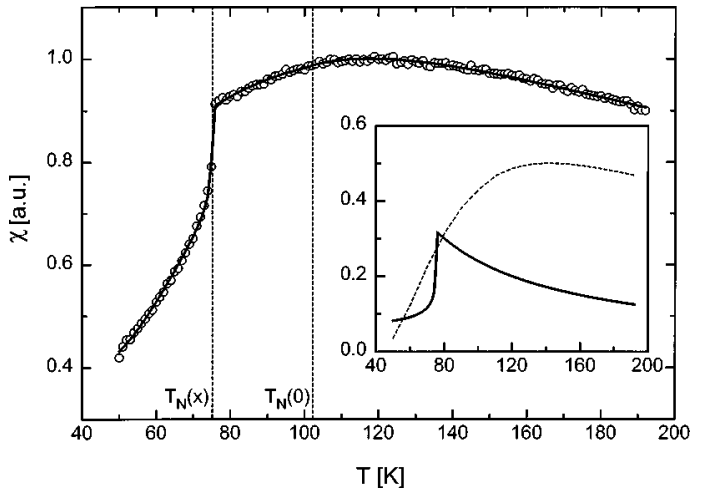

FIG. 2. Temperature dependence of the in-phase component of the complex zero-field susceptibility of $\mathrm{Rb}_{2} \mathrm{Co}_{0.85} \mathrm{Mg}_{0.15} \mathrm{~F}_{4}$ at constant frequency $f=10 \mathrm{~Hz}$. The full line represents the best fit of Eq. (11) to the data (see text). The dashed vertical lines mark the boundaries of the Griffiths phase $T_{N}(x)<T<T_{N}(0)$. The inset shows the decomposition of the fitting result into $\left\langle\chi_{\text {energy }}\right\rangle$ and $\left\langle\chi_{\text {random }}\right\rangle$ vs $T$ (broken and solid line, respectively).

squares). In contrast with that, the $\chi$ vs $T$ data of $\mathrm{Rb}_{2} \mathrm{Co}_{0.85} \mathrm{Mg}_{0.15} \mathrm{~F}_{4}$ (Fig. 2, open circles) exhibit a drastically steepened increase of $\chi$ at $T \gtrless T_{N}(x=0.15)$. It is followed by a quasilinear increase between $T_{N}(x)=75.2 \mathrm{~K}$ and $T_{N}(0)$ $=102 \mathrm{~K}$. Both features are significant deviations from the temperature behavior of the pure system. In contrast with Breed and co-workers, ${ }^{8}$ we measured the temperature dependence of the in-phase component $\chi^{\prime}$ of the complex ac susceptibility via superconducting quantum interference device (SQUID) susceptometry at constant frequency $f=10 \mathrm{~Hz}$. Since $f=10 \mathrm{~Hz}$ is far below the typical spin-flip frequency of $\approx 10^{10} \mathrm{~Hz}$ and magnetic losses are negligible, the in-phase component $\chi^{\prime}$ of the complex susceptibility is a good approximation of the static susceptibility $\chi$. Moreover, Ikeda obtained very similar results on $\mathrm{Rb}_{2} \mathrm{Co}_{0.8} \mathrm{Mg}_{0.2} \mathrm{~F}_{4}$ with static magnetometry. ${ }^{7}$ Hence, dynamical rounding of the data is ruled out.

With increasing dilution the evolution of the $\chi$ vs $T$ data proceeds in such a way that the steepened increase, which one observes for $x=0.15$, merges into a pronounced peak at $T_{N}(x)$ for $x=0.4$ (Fig. 3, open squares). For $T \ll T_{N}(x)$, the susceptibility increases again with decreasing temperature

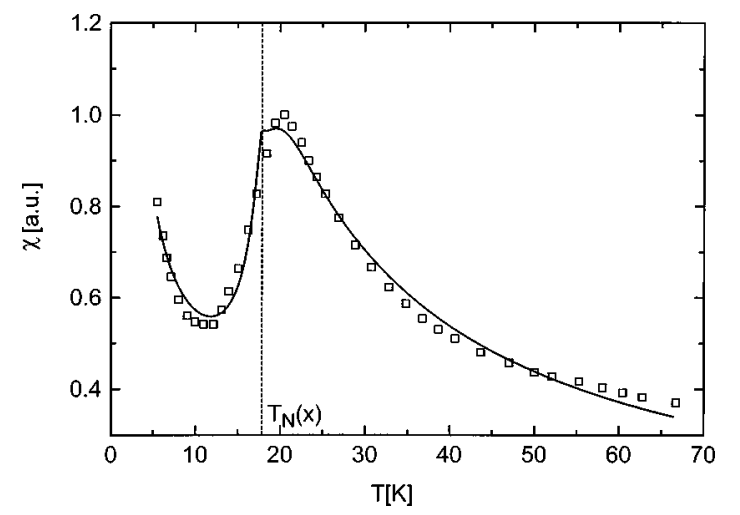

FIG. 3. Temperature dependence of the zero-field susceptibility $\chi$ (squares) of $\mathrm{Rb}_{2} \mathrm{Co}_{0.6} \mathrm{Mg}_{0.4} \mathrm{~F}_{4}$ (data from Ref. 7). The full line represents the best fit of Eq. (11) to the data (see text). $T_{N}(x)$ is indicated by a vertical dashed line. according to the paramagnetic response of isolated magnetic moments. The $\chi$ vs $T$ data in Fig. 3 were measured by Ikeda. ${ }^{7}$ He used SQUID magnetometry and the approximation $\chi \approx M / H$ with $H=0.057 \mathrm{MA} / \mathrm{m}(720 \mathrm{Oe})$.

\section{THEORY AND COMPARISON WITH EXPERIMENTAL RESULTS}

According to Fishman and Aharony ${ }^{5,6}$ the contribution of the singular part of the free energy to the static uniform susceptibility of a diluted Ising antiferromagnet is given by

$$
\chi\left(T, T_{N}\right)=\frac{1}{T}\left(A_{1}+A_{2}|t|^{1-\alpha}-A_{3}|t|^{2 \beta+\gamma-\phi}\right),
$$

where $t=\left(T-T_{N}\right) / T_{N}$ is the reduced temperature, $A_{1,2,3}$ are constants with $A_{2}(t>0) / A_{2}(t<0) \approx 1,{ }^{9} A_{3}(t>0) \equiv 0,{ }^{6}$ and $\alpha, \beta, \gamma$ are the critical exponents of the specific heat, the staggered magnetization, and staggered susceptibility, respectively. $\phi>\gamma$ is the crossover exponent from randomexchange to random-field critical behavior. It should be noticed that criticality is destroyed by arbitrarily weak external fields in $D=2$ dimensions as a consequence of the resulting random fields acting on the af order parameter. Nevertheless, the crossover exponent $\phi$ is well defined. ${ }^{6,9}$ As will be shown below, our analysis reveals $\phi \approx 1.85$, i.e., $\phi / \gamma \approx 1.06$ if $\gamma=1.75$ is accepted for the $2 \mathrm{D}$ random-exchange Ising model (REIM). ${ }^{6}$ It is the aim of the following analysis to generalize Eq. (1) in order to describe the global temperature dependence of $\chi$ in the concentration range $0 \leqslant x \leqslant 0.4$.

We start with generalizing the first $t$-dependent term of Eq. (1). It is called the energetic contribution of $\chi$ (Ref. 6) and is well known from the pure 2D Ising antiferromagnet. In that case, the specific heat $C$ is related to the susceptibility according to

$$
C \approx f \frac{d}{d T}(T \chi)
$$

where $f$ is a slowly varying function of temperature. ${ }^{2}$ Integration of Eq. (2) with respect to the temperature between the boundaries $T$ and infinity yields for $f \approx$ const

$$
\chi_{\text {energy }}=\frac{b}{T}\left(1+\frac{U(T)}{F}\right)
$$

where $F=f b$ and $b=\lim _{T \rightarrow \infty}(T \chi)$ are constants and $U(T)$ is the magnetic internal energy. For pure $\mathrm{Rb}_{2} \mathrm{CoF}_{4}, U(T)$ is exactly known from Onsager's solution of the 2D Ising model. ${ }^{10}$ Although $\alpha=0$ represents the uncertain case with respect to the Harris criterion, ${ }^{11}$ it turns out that, in the case of diamagnetic dilution, $\alpha$ does not experimentally deviate from zero. The predicted log-log-behavior of the specific heat $^{12}$ may play a role extremely close to the critical temperature. However, from an experimental point of view this is indistinguishable from pure behavior in the case of weak dilution. ${ }^{13,14}$ We shall henceforth assume that the wellknown set of critical exponents, $\alpha=0, \beta=0.125, \gamma=1.75$, also holds for the 2D REIM. In particular, one expects that 
the temperature dependence of the energetic contribution of the susceptibility of the diluted system follows at least qualitatively the behavior of the pure 2D Ising system. Onsager's exact expression of the magnetic internal energy reads

$$
U(T)=-J \operatorname{coth} \frac{2 J}{k_{B} T}\left[1+\frac{2}{\pi}\left(2 \tanh ^{2} \frac{2 J}{k_{B} T}-1\right) K_{1}(\kappa)\right],
$$

where $J$ is the exchange energy, $k_{B}$ is Boltzmann's constant, $K_{1}(\kappa)$ is the elliptic integral of the first kind and $\kappa$ $=2 \sinh \left(2 J / k_{B} T\right) / \cosh ^{2}\left(2 J / k_{B} T\right)$. In order to obtain a closed analytic expression, the elliptic integral may be approximated by

$$
K_{1}(\kappa) \cong \frac{\pi}{2}+6.43766\left(\frac{1}{\left(1-\kappa^{2}\right)^{2 \pi / 100}}-1\right),
$$

with a relative accuracy of better then $0.2 \%$. Equation (5) is an empirical function that interpolates between the numerical values of $K_{1}$ according to a least-squares fit. In particular, the limiting cases $K_{1}(\kappa=0)=\pi / 2$ and $K_{1}(\kappa \rightarrow 1) \rightarrow \infty$ are exactly fulfilled.

In the remainder of Eq. (1),

$$
\chi_{\text {random }}\left(T, T_{N}\right)=\frac{1}{T}\left(A_{1}-A_{3}|t|^{2 \beta+\gamma-\phi}\right),
$$

the $t$-dependent term appears only in diluted systems. It originates from the fact that the configurational average of the products of the thermal spin averages do not necessarily cancel on summing up all possible pairs of these averaged products. ${ }^{5}$ Since the thermal spin averages are zero above $T_{N}$, this random-exchange term contributes to $\chi$ only for $T$ $<T_{N}$. For $T \ll T_{N},|t|^{2 \beta+\gamma-\phi}$ is a slowly varying function of temperature with $|t|^{2 \beta+\gamma-\phi} \rightarrow 1$ for $T \rightarrow 0$. It is, hence, reasonable to assume the approximate validity of this term outside the critical region. This was approximately verified for the linear susceptibility of the layered 3D REIM system $\mathrm{Fe}_{1-x} \mathrm{Mg}_{x} \mathrm{Cl}_{2}, \quad x=0.3$, where a steep descent of $\chi$ below $T_{N}(x)$ due to the $|t|^{2 \beta+\gamma-\phi}$ term with $2 \beta+\gamma-\phi=0.62$ charaterizes the low- $T$ region. ${ }^{15}$

Similar behavior is found for $\mathrm{Rb}_{2} \mathrm{Co}_{1-x} \mathrm{Mg}_{x} \mathrm{~F}_{4}, x=0.15$ (Fig. 2) and 0.4 (Fig. 3), and will be analyzed below. Very clearly, however, the temperature dependence of $\chi$ strongly deviates from the expected energylike behavior also at $T$ $>T_{N}(x)$. For $x=0.15$ we find a weak, but finite convex curvature (Fig. 2), which becomes much stronger for $x=0.4$ (Fig. 3). Similarly, as observed in the case of $\mathrm{Fe}_{0.7} \mathrm{Mg}_{0.3} \mathrm{Cl}_{2}$, a broadened peak emerges just above $T_{N}(x) .{ }^{15}$ At this point we have to recall that the temperature range $T_{N}(x) \leqslant T \leqslant T_{N}(0)$ represents the Griffiths regime, ${ }^{3}$ which is characterized by a continuous series of weak singularities. They are due to "local" phase transitions that contribute to the susceptibility according to a distribution function $P\left(T_{N}^{\prime}\right)$, as was discussed recently for the case of the $3 d$ REIM $\mathrm{Fe}_{0.47} \mathrm{Zn}_{0.53} \mathrm{~F}_{2}$ in zero ${ }^{4}$ and nonzero external field. ${ }^{16}$ In the following we shall attempt to describe these extra contributions to $\chi$ by taking into account that all of them obey a temperature dependence following Eq. (1), where $t=T / T_{N}^{\prime}$ -1 with $T_{N}(x) \leqslant T_{N}^{\prime} \leqslant T_{N}(0)$ and the term $\left(A_{2} / T\right)|t|^{1-\alpha}$ is replaced by Eq. (3). The distribution of "local" critical tem- peratures originates from the corresponding statistical distribution of the diamagnetic dilution, which accompanies the process of ideal crystal growth. Hence, we introduce a distribution function $P\left(T_{N}^{\prime}\right) d T_{N}^{\prime}$, which describes the probability to find a local critical temperature within the interval $\left[T_{N}^{\prime}, T_{N}^{\prime}+d T_{N}^{\prime}\right]$. It is reasonable to assume that the fluctuations of the dilution are normally distributed around the average value $x$. In the limit $x \rightarrow 0$, the critical temperature of a 2D Ising system with nearest-neighbor interaction is a linear function of the dilution which is given by $T_{N}(x)=T_{N}(0)(1$ $-1.33 x) .{ }^{11}$ Hence, the local critical temperatures $T_{N}^{\prime}$ are also normally distributed around the average value $T_{N}(x)$, which characterizes the critical temperature of the global phase transition. Below that temperature the whole sample is involved in the process of long-range order. The distribution function is explicitly given by

$$
P\left(T_{N}^{\prime}\right)=\frac{1}{\sqrt{2 \pi} \sigma} e^{-\left(T_{N}(x)-T_{N}^{\prime}\right)^{2} / 2 \sigma^{2}},
$$

where $\sigma$ determines its width.

Since the SQUID measures the integral magnetic response of the sample, the measured susceptibility is given by the weighted average of all local contributions $\chi\left(T_{N}^{\prime}\right)$. Keeping in mind the onset of long-range order at $T<T_{N}$, the average is given by

$$
\langle\chi(T)\rangle=\int_{T_{N}(x)}^{T_{N}(0)} d T_{N}^{\prime} P\left(T_{N}^{\prime}\right) \chi\left(T, T_{N}^{\prime}\right) .
$$

The local susceptibility contributions are given by the superposition of $\chi_{\text {energy }}$, Eq. (3), and $\chi_{\text {random }}$, Eq. (6). The fact that $P\left(T_{N}^{\prime}\right) \chi\left(T, T_{N}^{\prime}\right)$ is centered around some maximum value suggests to approximate Eq. (8) by saddlepoint integration methods. ${ }^{17}$ Within reasonable approximations (see Appendix A) straightforward calculation yields the following expression for the energetic contribution to Eq. (8),

$$
\begin{aligned}
\left\langle\chi_{\text {energy }}(T)\right\rangle= & \frac{a\left(1-\frac{U(T)}{F}\right)_{T_{N}=T_{M}(x)}}{T \sqrt{\left(\frac{T_{M}-T_{N}(x)}{\sigma^{2}}\right)^{2}+\frac{1}{\sigma^{2}}}} \\
& \times e^{-\left[T_{N}(x)-T_{M}\right]^{2} / 2 \sigma^{2}} \\
& \times\left(\sqrt{1-e^{-\sqrt{2}\left[T_{N}(0)-T_{M}\right]^{2}\left[\left(\left(T_{M}-T_{N}(x)\right) / \sigma^{2}\right)^{2}+1 / \sigma^{2}\right]}}\right. \\
& \left.-\sqrt{1-e^{-\sqrt{2}\left(T_{N}(x)-T_{M}\right)^{2}\left[\left(\left(T_{M}-T_{N}(x)\right) / \sigma^{2}\right)^{2}+1 / \sigma^{2}\right]}}\right),
\end{aligned}
$$

where $a$ is a proportionality constant, while $U(T)$ and $T_{M}(T)$ are given by Eqs. (4) and (A3), respectively. The exchange energy $J$, which enters the internal magnetic energy $U(T)$, is related to $T_{M}$ via $|J|=c_{3} T_{M}$ in order to take into account the proportionality between $J$ and the critical temperature. The proportionality constant $c_{3}$ is, however, expected to deviate from the value of the pure system, because in the case of a diluted system $J$ has the meaning of an 
effective exchange within a virtual crystal approximation. Hence, $c_{3}$ enters Eq. (4) as an unknown fitting parameter.

The above procedure of calculating $\left\langle\chi_{\text {energy }}\right\rangle$ applies similarly to the calculation of $\left\langle\chi_{\text {random }}\right\rangle$. Since
$\left(-A_{3} / T\right)|t|^{2 \beta+\gamma-\phi} \equiv 0$ for $T>T_{N}^{\prime}$, its averaging with respect to $P\left(T_{N}^{\prime}\right)$ has to be done subsequently for the temperature regions $T<T_{N}(x), T_{N}(x)<T<T_{N}(0)$, and $T>T_{N}(0)$. Straightforward calculation (see Appendix B) yields

$$
\left\langle\chi_{\text {random }}\right\rangle=\frac{k}{T}\left\{\begin{array}{ll}
I_{1}-c_{4} I_{2}\left[l=T_{N}(x), u=T_{N}(0)\right] & \text { if } T<T_{N}(x) \\
I_{1}-c_{4} I_{2}\left[l=T, u=T_{N}(0)\right] & \text { if } T_{N}(x) \leqslant T \leqslant T_{N}(0) \\
I_{1} & \text { if } T>T_{N}(0)
\end{array}\right\},
$$

with $c_{4}=A_{3} / A_{1}$ and $I_{1}, I_{2}(l, u)$ given by Eqs. (B2) and (B8), respectively.

In the final expression of the susceptibility

$$
\chi=\chi_{0}+\chi_{p} / T+\left\langle\chi_{\text {energy }}\right\rangle+\left\langle\chi_{\text {random }}\right\rangle,
$$

we have to take into account two correction terms that are not included in the above analysis. $\chi_{0}$ takes into account the background of the van Vleck type. It amounts to $\frac{1}{3}$ of the total parallel susceptibility in the case of the pure system ${ }^{8}$ (see Fig. 1). $\chi_{p} / T$ takes into account the paramagnetic susceptibility of isolated magnetic moments. Their influence increases with increasing dilution owing to the increasing concentration ratio of isolated to exchange coupled magnetic moments.

Figure 1 (solid line) shows the best fit of Eq. (11) to the $\chi$ vs $T$ data of pure $\mathrm{Rb}_{2} \mathrm{CoF}_{4}$. Clearly in this case $\left\langle\chi_{\text {random }}\right\rangle$ and $\chi_{p} / T$ vanish for obvious reasons. Further, $\left\langle\chi_{\text {energy }}\right\rangle$ degenerates to Eq. (3). The final result of the fitting procedure yields $b=116.604 \mathrm{~K}, 1 / F=0.616, J / k_{B}=92.92 \mathrm{~K}$, and $\chi_{0}=0.61$. Obviously, the data are well described by Eq. (11) within a wide temperature range. For the pure 2D Ising antiferromagnet on a square lattice the exact relation $T_{N}$ $=0.5673 q \mathrm{~J} / 2 k_{B}$ holds, ${ }^{1}$ where $q=4$ is the number of magnetic nearest neighbors. Using this relation we obtain $T_{N}$ $=105.4 \mathrm{~K}$. Obviously, this relation also holds for our "energetic" approximation, because the derivative $d / d T(T \chi)$ vs $T$ of the fitting result (Fig. 1, broken line) exhibits a virtually logarithmic singularity at $T_{N}(0)=105.4 \mathrm{~K}$, which deviates from the experimental value $T_{N}(0) \approx 102 \mathrm{~K}$ (Fig. 1, squares) by only $\approx 3 \%$. Although this discrepancy may origin from the inadequate temperature resolution of the experimental data in the vicinity of $T_{N}$, a systematic shift of the fitting result cannot be ruled out according to the weak, but finite Heisenberg-type character of the exchange interaction in $\mathrm{Rb}_{2} \mathrm{CoF}_{4}{ }^{18}$ However, the large value of the background $\chi_{0}=0.61$ remains unreasonable. It may indicate that the approximation $\chi \approx M / H$ is not appropriate in the case of $H$ $=0.77 \mathrm{MA} / \mathrm{m}$ used in the experiments. ${ }^{8}$ Probably, the applied magnetic field is not small enough in order to avoid nonlinearities of $M$ vs $H$.

Figure 2 (solid line) shows the result of the best fit of Eq. (11) to the $\chi$ vs $T$ data of $\mathrm{Rb}_{2} \mathrm{Co}_{0.85} \mathrm{Mg}_{0.15} \mathrm{~F}_{4}$. With regard to the relatively high temperatures, $T>50 \mathrm{~K}$, the paramagnetic contribution $\chi_{p} / T$ has been neglected. The fit involves the proportionality constants $a=384.06$ and $k=90.73$ that enter
Eqs. (9) and (10), respectively. Moreover, the fitting procedure yields the values of $1 / F=0.049$ that enters Eq. (1), $c_{2}=132.24$ that enters Eq. (A3), $c_{3}=1.19$ that mediates between the effective exchange, and $T_{M}, c_{4}=0.483$ that enters Eq. (10), the van Vleck background $\chi_{0}=0.31$ (Ref. 8) and $\sigma=0.38$ the width of the distribution function $P\left(T_{N}^{\prime}\right)$. Furthermore we find $2 \beta+\gamma-\phi=0.14$, which implies $\phi=1.86$. Hence, $\phi / \gamma=1.06$ and $2 \beta+\gamma-\phi=0.14<2 \beta=0.25$ is in accordance with theory. ${ }^{6}$ The inset of Fig. 2 shows the energetic (dashed line) and the random exchange contribution (solid line), respectively. As expected, the steep increase of $\chi$ vs $T$ below $T_{N}(x)$ originates exclusively from the nonenergetic contribution $\left\langle\chi_{\text {random }}\right\rangle$. The quasilinear increase of $\chi$ vs $T$ within $T_{N}(x)<T<T_{N}(0)$ appears to be accidental in view of the opposite curvatures revealed by both contributions. Hence, this remarkable temperature dependence cannot be taken as a typical signature of the Griffiths phase as might have been assumed at the first glance. However, since the Griffiths temperature $T_{N}(0)$ enters our theory, Eqs. (9) and (10), it is at the origin of a subtle kinklike temperature dependence of $d \chi / d T$ vs $T$ at $T_{N}(0)$. In fact, closer inspection of the temperature derivative of the experimental data seems to confirm this prediction, although the scatter of the data prevents its ultimate evidence.

Figure 3 (solid line) shows the best fit of Eq. (11) to the $\chi$ vs $T$ data of $\mathrm{Rb}_{2} \mathrm{Co}_{0.6} \mathrm{Mg}_{0.4} \mathrm{~F}_{4}$. In this case the energetic contribution to $\langle\chi\rangle$ turns out to be negligible with respect to the random-exchange contribution. The fitting procedure yields $k=6.60, \quad c_{4}=0.68, \quad \chi_{p}=7.46, \quad T_{N}(x)=17.79 \mathrm{~K}, \quad$ and $\chi_{0}$ $=0.04$. Very remarkably, we find $2 \beta+\gamma-\phi=0.13$, which differs but slightly from the corresponding fitting parameter for $x=0.15$. Again, the condition $2 \beta+\gamma-\phi=0.13<0.25$ is fulfilled. As expected, the resulting width $\sigma=2.66$ of the distribution function is larger than for $x=0.15$. The observed amount, however, $\sigma(0.4) \approx 7.1 \sigma(0.15)$, does not follow the relation $\Delta x \propto \sqrt{x}$, which one might expect from natural statistics. Probably this is a consequence of the crossover from equilibrium (Gaussian) to percolation (Poisson) statistics when approaching the percolation threshold, $x_{p}=0.41$. At closer inspection of Fig. 3 the fit exhibits a kinklike behavior at $T_{N}(x)$. According to our theory, this reflects the residual influence of the temperature dependence of the thermal spin averages, which vanish at $T>T_{N}^{\prime}$. Unfortunately the temperature resolution of the experimental data is not sufficient in order to resolve this detailed structure. 


\section{CONCLUSION}

Although diamagnetic dilution does virtually not affect the critical behavior of 2D Ising systems, there are strong qualitative deviations of the uniform magnetic susceptibility $\chi$ of $\mathrm{Rb}_{2} \mathrm{Co}_{1-x} \mathrm{Mg}_{x} \mathrm{~F}_{4}$ from the "energetic" $\chi$ vs $T$ behavior of the pure compound, $x=0$. First, owing to subtractive random-exchange extra contributions, $\left\langle\chi_{\text {random }}\right\rangle$, we observe a steep decrease of $\chi$ vs $T$ just below $T_{N}(x)$. Second, Griffiths-type weak singularities have to be taken into account at temperatures between $T_{N}(x)$ and $T_{N}(0)$. They are composed of contributions due to both $\chi_{\text {energy }}$ and $\chi_{\text {random }}$ that form a flat, nearly linear anomaly in the case $x=0.15$. For higher dilution, $x=0.4$, the contributions due to $\chi_{\text {random }}$ give rise to a pronounced peak at $T_{N}(x)$.

Originally, $\mathrm{Ikeda}^{7}$ proposed a coupling mechanism between the uniform and the staggered susceptibility in order to explain this peak structure. However, within this paper we have shown, that the dilution-induced evolution of the peak structure in $\chi$ vs $T$ originates from the crossover from pure Ising to random-exchange dominated behavior. Since this crossover affects also the temperature dependence of $\chi$ outside the critical region, we have generalized Aharony's expression of $\chi$ for REIM antiferromagnets ${ }^{6}$ in a phenomenological way. This generalized expression has then to be averaged with respect to the distribution of local critical temperatures, which are distributed throughout the temperature interval $T_{N}(x)<T_{N}^{\prime}<T_{N}(0)$ of the Griffiths phase. Within the framework of an averaged and generalized susceptibility function we are thus able to model the temperature dependence of the susceptibility of $\mathrm{Rb}_{2} \mathrm{Co}_{1-x} \mathrm{Mg}_{x} \mathrm{~F}_{4}$ for the concentrations $x=0,0.15$, and 0.4 , i.e., throughout the concentration range in which long-range order is exhibited. For $x$ $=0.15$ the susceptibility mediates between two extreme scenarios, the pure Ising behavior $(x=0)$, which is characterized by the energetic susceptibility $\left\langle\chi_{\text {energy }}\right\rangle$, and the REIM behavior, which is dominated by $\left\langle\chi_{\text {random }}\right\rangle$ in the limit of strong dilution, $x=0.4$.

It will be interesting to consider the 3D REIM case in an analogous way. Data obtained previously on $\mathrm{Fe}_{0.7} \mathrm{Mg}_{0.3} \mathrm{Cl}_{2}$ (Ref. 15) seem to hint at strong importance of $\left\langle\chi_{\text {random }}\right\rangle$, which dominates not only at $T<T_{N}$, but also in the peak region just above $T_{N}$. Analysis will, however, be more tedious than in the above $2 \mathrm{D}$ case, since the pure-to-random crossover in $D=3$ dimensions is connected with a change of the critical behavior. This rules out, e.g., a simple approximation of the energylike contribution of the susceptibility.

\section{ACKNOWLEDGMENTS}

Financial support by the Deutsche Forschungsgemeinschaft is gratefully acknowledged. D. P. Belanger also acknowledges support from DOE Grant No. DE-FG0387ER45324.

\section{APPENDIX A}

We start with the saddlepoint integration of $\left\langle\chi_{\text {energy }}\right\rangle$. According to Eq. (7) the temperature-dependent position $T_{M}$ of the maximum value of $P\left(T_{N}^{\prime}\right) \chi\left(T_{N}^{\prime}\right)$ can be determined from the following equation:

$$
\left(\frac{\partial \chi}{\partial T_{N}^{\prime}}\right)_{T_{M}}=\chi\left(T_{M}\right) \frac{T_{M}-T_{N}(x)}{\sigma^{2}} .
$$

From that we calculate $T_{M}=T_{M}(T)$ while we restrict ourselves for simplicity to the approximation

$$
\chi\left(T_{N}^{\prime}\right)=\frac{c_{1}}{T}\left[1-c_{2}\left(1-\frac{T_{N}^{\prime}}{T}\right) \ln \left|1-\frac{T_{N}^{\prime}}{T}\right|\right],
$$

which reproduces the temperature behavior of Eq. (2) in the critical region $T \approx T_{N}^{\prime} \cdot{ }^{1}$ Although $c_{1}$ and $c_{2}$ are known in the case of the square Ising lattice, $c_{2}$ becomes a free parameter within the following analysis in order to compensate the deviations of Eq. (A2) with respect to Eq. (3) outside the critical region. In the limit $T \approx T_{M}$ the approximation $\left(1-T_{M} / T\right) \ln \left|1-T_{M} / T\right| \approx 0$ holds. Insertion of Eq. (A2) into Eq. (A1) and using the above approximation yields

$$
T_{M}=T_{N}(x)+\frac{c_{2} \sigma^{2}}{T} .
$$

A series expansion of $\chi\left(T_{N}^{\prime}\right)$ and $P\left(T_{N}^{\prime}\right)$ up to first and second order in powers of $\left(T_{N}^{\prime}-T_{M}\right)$, respectively, yields

$$
\chi\left(T_{N}^{\prime}\right) P\left(T_{N}^{\prime}\right) \approx A+\frac{B}{2}\left(T_{N}^{\prime}-T_{M}\right)^{2}
$$

with $A=\chi\left(T_{M}\right) P\left(T_{M}\right)$ and $B=-A\left[\left(\left(T_{M}-T_{N}(x)\right) / \sigma^{2}\right)^{2}\right.$ $\left.+1 / \sigma^{2}\right]$. The linear term of the expansion vanishes due to Eq. (A1). With Eq. (A4) we calculate the weighted average of $\chi_{\text {energy }}$ within the saddle-point approximation. It reads

$$
\left\langle\chi_{\text {energy }}\right\rangle \propto A \int_{T_{N}(x)}^{T_{N}(0)} d T_{N}^{\prime} e^{(-|B| / A)\left(T_{N}^{\prime}-T_{M}\right)^{2}} .
$$

Since $\exp \left[-|B|\left(T_{N}^{\prime}-T_{M}\right)^{2} / A\right]$ does not vanish at $T_{N}(x)$ and $T_{N}(0)$, it is not appropriate to shift the boundaries of the integration towards infinity. Hence, in order to calculate the Gaussian integral with finite boundaries, we use the approximation

$$
\int_{0}^{a} d t e^{-t^{2}} \approx \frac{1}{2} \sqrt{\int_{0}^{\bar{r}} d r \int_{0}^{2 \pi} d \varphi r e^{-r^{2}}}=\frac{1}{2} \sqrt{\pi\left(1-e^{-\bar{r}^{2}}\right)},
$$

where $\bar{r}=a 2^{1 / 4}$ is the geometrical average of the radius of the largest inner and the smallest outer circle that fills in and contains a square of the area $4 a^{2}$, respectively. Within this approximation Eq. (A5) yields the explicit result of Eq. (9).

\section{APPENDIX B}

In the case $T<T_{N},\left\langle\chi_{\text {random }}\right\rangle$ is given by

$$
\begin{aligned}
& \left\langle\chi_{\text {random }}\right\rangle \propto \frac{A_{1}}{T}\left\{\int_{T_{N}(x)}^{T_{N}(0)} d T_{N}^{\prime} e^{-\left[T_{N}^{\prime}-T_{N}(x)\right]^{2} / 2 \sigma^{2}}\right. \\
& \left.-c_{4} \int_{T_{N}(x)}^{T_{N}(0)} d T_{N}^{\prime}\left|\frac{T-T_{N}^{\prime}}{T_{N}^{\prime}}\right|^{2 \beta+\gamma-\phi} e^{-\left[T_{N}^{\prime}-T_{N}(x)\right]^{2} / 2 \sigma^{2}}\right\}
\end{aligned}
$$


with $c_{4}=A_{3} / A_{1}$. Using approximation (A6) the first integral yields

$$
\begin{aligned}
I_{1}: & =\int_{T_{N}(x)}^{T_{N}(0)} d T_{N}^{\prime} e^{-\left[T_{N}^{\prime}-T_{N}(x)\right]^{2} / 2 \sigma^{2}} \\
& \cong \sqrt{\frac{\pi}{2}} \sigma \sqrt{\left(1-e^{-\left[T_{N}(0)-T_{N}(x)\right]^{2} / \sqrt{2} \sigma^{2}}\right)} .
\end{aligned}
$$

The solution of the second integral via saddle-point integration requires the calculation of the maximum position according to $d / d T_{N}^{\prime}\left[\left|\left(T-T_{N}^{\prime}\right) / T_{N}^{\prime}\right|^{2 \beta+\gamma-\phi} P\left(T_{N}^{\prime}\right)\right]_{\mid T_{N}^{\prime}=T_{M}}=0$. Taking into account $T<T_{N}^{\prime}$, one obtains

$$
\left[T_{M}-T_{N}(x)\right]\left(T_{M}-T\right) T_{M}=(2 \beta+\gamma-\phi) T \sigma^{2} .
$$

In order to obtain a quadratic equation in $T_{M}$, Eq. (B3) is roughly simplified within the approximation $T_{M} \approx T_{N}(x)$. It yields

$$
\left[T_{M}-T_{N}(x)\right]\left(T_{M}-T\right) T_{N}(x)=(2 \beta+\gamma-\phi) T \sigma^{2},
$$

which has the solution

$$
\begin{aligned}
T_{M}^{\mathrm{random}}= & \frac{1}{2}\left(\left[T_{N}(x)+T\right]\right. \\
& \left.+\sqrt{\left[T_{N}(x)-T\right]^{2}+4(2 \beta+\gamma-\phi) \sigma^{2} \frac{T}{T_{N}(x)}}\right) .
\end{aligned}
$$

Since $T_{M}^{\text {random }}$ is located within the interval $T_{N}(x)<T_{M}^{\mathrm{random}}<T_{N}(0)$, the corresponding negative solution of Eq. (B5) has to be discarded.

The series expansion of $\left|\left(T-T_{N}^{\prime}\right) / T_{N}^{\prime}\right|^{2 \beta+\gamma-\phi} \exp \left\{-\left[T_{N}^{\prime}\right.\right.$ $\left.\left.-T_{N}(x)\right]^{2} / 2 \sigma^{2}\right\}$ up to the second order in powers of $\left(T_{N}^{\prime}\right.$ $-T_{M}^{\mathrm{random}}$ ) yields for $T<T_{N}(x)$,

$$
\begin{aligned}
\left\langle\chi_{\text {random }}\right\rangle \propto & \frac{A_{1}}{T}\left(I_{1}-c_{4} A_{\text {random }}\right. \\
& \left.\times \int_{T_{N}(x)}^{T_{N}(0)} d T_{N}^{\prime} e^{-\left(\left|B_{\text {random }}\right| / A_{\text {random }}\right)\left(T_{N}^{\prime}-T_{M}^{\text {random }}\right)^{2}}\right)
\end{aligned}
$$

with $A_{\text {random }}=\sqrt{2 \pi} \sigma\left(1-T / T_{M}^{\mathrm{random}}\right)^{2 \beta+\gamma-\phi} P\left(T_{M}^{\mathrm{random}}\right)$ and

$$
\begin{aligned}
B_{\text {random }}= & -A_{\text {random }}\left((2 \beta+\gamma-\phi) T \frac{2 T_{M}^{\text {random }}-T}{\left[T_{M}^{\text {random }}\left(T_{M}^{\text {random }}-T\right)\right]^{2}}\right. \\
& \left.+\frac{1}{\sigma^{2}}\right),
\end{aligned}
$$

while $I_{1}$ is given by Eq. (B2). In order to obtain an explicit expression of the second integral that enters the proportionality (B6), we calculate

$$
I_{2}(l, u):=A_{\text {random }} \int_{l}^{u} d T_{N}^{\prime} e^{-\left(\left|B_{\text {random }}\right| / A_{\text {random }}\right)\left(T_{N}^{\prime}-T_{M}^{\text {random }}\right)^{2}}
$$

using generalized lower and upper boundaries $l$ and $u$. Insertion of $A_{\text {random }}$ and $B_{\text {random }}$ into Eq. (B7) and application of the approximation (A6) yields

$$
\begin{aligned}
I_{2}(l, u)= & \frac{\pi \sigma}{\sqrt{2}} \frac{\left(1-T / T_{M}^{\mathrm{random}}\right)^{2 \beta+\gamma-\phi} P\left(T_{M}^{\mathrm{random}}\right)}{\sqrt{(2 \beta+\gamma-\phi) T \frac{2 T_{M}^{\mathrm{random}}-T}{\left[T_{M}^{\mathrm{random}}\left(T_{M}^{\mathrm{random}}-T\right)\right]^{2}}+\frac{1}{\sigma^{2}}}} \\
& \times\left\{\sqrt{1-e^{-\sqrt{2}\left\{[(2 \beta+\gamma-\phi) T]\left(2 T_{M}^{\mathrm{random}}-T\right) /\left[T_{M}^{\mathrm{random}}\left(T_{M}^{\mathrm{random}}-T\right)\right]^{2}+1 / \sigma^{2}\right\}\left(u-T_{M}^{\text {random }}\right)^{2}}}\right. \\
& -\sqrt{\left.1-e^{-\sqrt{2}\left\{[(2 \beta+\gamma-\phi) T]\left(2 T_{M}^{\text {random }}-T\right) /\left[T_{M}^{\text {random }}\left(T_{M}^{\text {random }}-T\right)\right]^{2}+1 / \sigma^{2}\right\}\left(l-T_{M}^{\text {random }}\right)^{2}}\right\} .}
\end{aligned}
$$

In the case $T_{N}(x)<T<T_{N}(0),\left\langle\chi_{\text {random }}\right\rangle$ is given by

$$
\left\langle\chi_{\text {random }}\right\rangle \propto \frac{A_{1}}{T}\left\{I_{1}-c_{4} I_{2}\left[l=T, u=T_{N}(0)\right]\right\},
$$

according to $\left(-A_{3} / T\right)|t|^{2 \beta+\gamma-\phi} \equiv 0$ for $T>T_{N}^{\prime}$. In the case $T>T_{N}(0)$, the second term of Eq. (B9) vanishes.

${ }^{1}$ M. F. Sykes and M. E. Fisher, Physica (Amsterdam) 28, 919 (1962)

${ }^{2}$ M. E. Fisher, Philos. Mag. 7, 1731 (1962).

${ }^{3}$ R. B. Griffiths, Phys. Rev. Lett. 23, 17 (1969).

${ }^{4}$ Ch. Binek and W. Kleemann, Phys. Rev. B 51, 12888 (1995).
${ }^{5}$ S. Fishman and A. Aharony, J. Phys. C 12, L729 (1979).

${ }^{6}$ A. Aharony, Europhys. Lett. 1, 617 (1986).

${ }^{7}$ H. Ikeda, J. Phys. C 16, L21 (1983).

${ }^{8}$ D. J. Breed, K. Gilijamse, and A. R. Miedema, Physica (Amsterdam) 45, 205 (1969). 
${ }^{9}$ D. P. Belanger, Braz. J. Phys. 22, 283 (1992).

${ }^{10}$ K. Huang, Statistical Mechanics Vol. III (Wiley, New York, 1963).

${ }^{11}$ A. B. Harris, J. Phys. C 7, 1671 (1974).

${ }^{12}$ V. S. Dotsenko and V. S. Dotsenko, J. Phys. C 15, 495 (1982).

${ }^{13}$ H. Ikeda, J. Phys. Soc. Jpn. 50, 3215 (1981).

${ }^{14}$ I. B. Ferreira, A. R. King, V. Jaccarino, J. L. Cardy, and H. J. Guggenheim, Phys. Rev. B 28, 5192 (1983).
${ }^{15}$ U. A. Leitão and W. Kleemann, Europhys. Lett. 5, 529 (1988).

${ }^{16}$ Ch. Binek, S. Kuttler, and W. Kleemann, Phys. Rev. Lett. 75, 2412 (1995).

${ }^{17}$ E. Madelung, Die Mathematischen Hilfsmittel des Physikers, Vol. 4 (Springer-Verlag, Berlin, 1964).

${ }^{18}$ P. Nordblad, D. P. Belanger, A. R. King, and V. Jaccarino, Phys. Rev. B 28, 278 (1983). 